\title{
Open call for papers: COVID-19 pandemic
}

\section{With a rapid publication process}

\section{Special Call for Peer Reviewers}

The HPCDP Journal is currently seeking volunteer peer reviewers with interdisciplinary expertise to conduct timely reviews of manuscripts submitted to the journal through the issued open call for papers on the COVID-19 pandemic and its links to the fields of health promotion and chronic disease prevention.

Relevant topic areas include, but are not limited to:

- Chronic diseases, their risk factors and links with COVID-19 (e.g., increased risk of severe illness and longer term health effects)

- Mental health

- $\quad$ Problematic substance use

- Impact and consequences of public health measures

- Delivery of preventive health care

- Emerging evidence for promising interventions

- Health equity

To be considered as a potential peer reviewer for the HPCDP Journal COVID-19 series, please email us at PHAC.HPCDP.Journal-Revue .PSPMC.ASPC@canada.ca, and briefly indicate your areas of expertise, institutional affiliation(s) and availability. A concise biosketch, C.V. or biolinks may be provided to assist in the selection process.

Reviewers are asked to commit to completing at least one peer review within 5 days of accepting a request.

The societal impact of the novel coronavirus disease (COVID-19) pandemic is multifaceted, and all Canadians, one way or another, have been affected. From a public health perspective, we also see this pandemic colliding with the slow-motion chronic disease epidemic that is affecting all parts of the globe.

Health Promotion and Chronic Disease Prevention in Canada: Research, Policy and Practice (the HPCDP Journal) is the monthly, online scientific journal of the Health Promotion and Chronic Disease Prevention Branch of the Public Health Agency of Canada. The HPCDP Journal is hereby inviting original quantitative and qualitative research papers, commentaries, editorials and At-a-glance manuscripts that address the links between the COVID-19 pandemic and health promotion, chronic disease and health equity.

There are many relevant topics, including, but not limited to:

- $\quad$ Associations between chronic diseases (and their risk factors) and the risk for infection, severe illness and poorer outcomes.

- The longer-term health effects of COVID-19 on survivors, including long-lasting mental health issues such as depression, anxiety and more.

- $\quad$ Studying the public health response and its impact and unintended consequences at the individual level (e.g. physical and mental health, health and health-seeking behaviours), family level, and the community or societal level.

- $\quad$ The delivery of preventive health care during the pandemic.

- Emerging scientific evidence, including through natural experimental studies, about promising interventions to improve the public health response (e.g. social distancing measures, protecting people with underlying chronic conditions) or to mitigate the negative impacts of the response (e.g. mental health consequences).

- Health equity and the social determinants of health as cross-cutting issues. 
To ensure lasting relevance, we expect all submissions to discuss the implications of their findings for the recovery phase of the current crisis, and beyond.

Manuscripts will be considered as they are received. Those selected for further consideration will be assigned to a special editorial committee dedicated to this series, as well as to two peer reviewers if appropriate for the article type.

We will strive to provide an initial editorial decision on submitted manuscripts within 15 business days of completed submission for peer-reviewed papers and five business days for non-peer-reviewed manuscripts. Accepted manuscripts will be prioritized for publication and will appear online, in HTML format, and be indexed as "ahead of print" articles prior to being produced in PDF and included in a regular issue of the Journal.

Refer to our website for information on invited article types and detailed submission guidelines for authors: https://www.canada.ca /en/public-health/services/reports-publications/health-promotion-chronic-disease-prevention-canada-research-policy-practice /information-authors.html.

For any pre-submission questions about suitability or scope, please direct inquiries to PHAC.HPCDP.Journal-Revue.PSPMC.ASPC@canada.ca.

Submission information: Kindly refer to this call for papers in your submission covering letter and submit manuscripts by email to PHAC.HPCDP.Journal-Revue.PSPMC.ASPC@canada.ca. This call will continue until further notice.

Submission deadline: Open until further notice. 